\title{
Visual beats: Phenomenology and preliminary data as a function of age'
}

\section{RATHE KARRER, ILLINOIS STATE PEDIATRIC INSTITUTE, Chicago, Ill. 60608}

Subjects, ranging in Age from eight to 71 , observed and described visual beats arising from binocular and dichoptic presentation of two different flicker frequencies. Beat characteristics were strikingly similar in the two conditions and over age.

Although beats in audition have been extensively studied since 1700 (von Békésy, 1960; Wever, 1929), they have been neglected in vision. This neglect is probably rooted in the essential differences between auditory and visual stimuli and between the two receptors. For the production of perceptible visual beats relatively slow frequencies obtainable by pulsing light are necessary. With pulsed light the situation is not one of addition and subtraction of two different frequencies as with auditory beats. The total light from two different frequencies may be distributed in time as the pulses are maximally out-of-phase, or condensed in time as the pulses become in-phase.

Schwarz (1943) described visual beats as a change in brightness and frequency of the flicker while Attneave \& McReynolds (1950) observed changes in flicker rate and a phi-like movement from one source to the other when in binocular regard. Clausen \& Vanderbilt (1957) superimposing photic and electric flicker frequencies emphasized a compelling horizontal apparent movement of light within the field (see Karrer, 1965, for review of beat studies).

Prior to further investigation of visual beats 1 felt it necessary to further study their phenomenology in both binocular and dichoptic viewing conditions. In the binocular condition the beat can result from the physical interaction of frequencies prior to perception. In the dichoptic condition the beat must arise from the neural counterparts of the two frequencies interacting in the visual system. This report presents these descriptions as a function of age.

Methods and Procedure. Flicker of 20 and $21 \mathrm{pps}$ produced by two General Radio Strobotacs (Model 1531 A) served as stimuli (see Karrer, 1967, for details of apparatus). On separate days descriptions for the binocular and dichoptic conditions were obtained from each S. Because of the greater difficulty in observing dichoptic beats the binocular condition was always first. Generally, instructions to look for and describe the beat rhythm, as well as whatever else came to his attention, yielded rather detailed descriptions. After describing the beat the $S$ tapped in synchrony with it so that beat perception could be objectively verified. Simple visual inspection of the alignment of tap and beat on polygraph records established the synchrony of the S's tapping. Each S's eye dominance was determined with the Miles $A B C$ test and by the sighting of a toy rifle and telescope.

The study utilized five $S s$ at each of five age levels: 8-10, 12-14, 18-24, $30-40$, and 50-71. Two Ss in each group were female. There were no visual abnormalities or severe impairment of acuity in any of the Ss.

Results. Twenty-four of the $25 \mathrm{Ss}$ were able to perceive the binocular beats while 22 Ss perceived the dichoptic beats. One $S$ in the $8-10$ group and two $S$ s in the $50-71$ group were unable to see a dichoptic beat. The dichoptic condition was exceedingly difficult. The beat was faint and slight eye movements or changes in attentional foci invariably resulted in loss of the phenomenon. Steady concentration of attention and fixation was a necessity. Descriptions of the beat were surprisingly uniform and similar in both conditions; a few characteristics being almost always present. In order of their prevalence they are: (1) movement, (2) brightness change, (3) pulsation, (4) flicker change, (5) line patterns, and (6) color change ${ }^{2}$. Only three Ss (binocular) reported seeing all six; usually only three to five were reported. There was a tendency to report fewer characteristics in the dichoptic condition.

The most striking and prevalent description was one of slow motion. This motion appeared either as a bright spot, a grid like pattern, radiating lines, or as an expanding and contracting purplish spot which moved toward and away from the observer. Quite of ten the movement appeared as a wave like motion of some rather fuzzy brightness differential sweeping across the field and back or travelling in three-dimensional, elliptical rotation. Rhythmical brightness changes usually appeared in the center of the field of ten giving rise to pulsatory movement analogous to gamma. Described as a momentary "stopping" of the flicker, the flicker change usually occurred as the field became brighter and sometimes as the whole field appeared to come toward the $S$. The line patterns frequently appeared as a "grid" or as radiating lines from the center. The grid-like patterns seen toward the center of the field became brighter and larger, while they occasionally moved from side to side or from front to rear, with the beat rhythm. In contrast, the radiating line patterns seen toward the periphery became more intense or larger with the beat. Occasionally, color was associated with the beat, even in the dichoptic beat. The vertical grid was alternately red and green or the cells were different varying colors. Color changes were the least reported and were generally described (binocular beat) as a purple spot expanding and contracting or (dichoptic beat): as little bluish spots running across the field, as an eccentric purple spot that rotated, or as a purple line that moved downward and then disappeared, only to reappear for a new cycle.

The beat was localized in any part of the field. Eye dominance played no strong role as it was unrelated to how or where the beat was perceived. Even though the nondominant visual field was partially supressed in dichoptic viewing, its flicker frequency interacted with the frequency in the dominant field and produced beats.

There was little difference in the observations of the age groups although the oldest group did have one $S$ reporting no binocular beat and two Ss reporting no dichoptic beat. They also tended to give fewer descriptions involving flicker change and pulsation. Neither were there any indications of consistent sex differences.

Discussion. While all of the characteristics, both binocular and dichoptic, were never seen simultaneously, movement, brightness change, and patterning often combined into a clear rhythmical experience. Flicker change, patterning, and color change similarly combined into a unified percept. When the $S$ was attending to movement effects, he found it difficult to be aware of the flicker change with the converse also true. Some of these effects are present to a certain extent in flicker alone. Patterning, expansive and contractive movement, brightness change, and even color have been known to occur from a single flicker source since Purkinje. In the beat condition these phenomena are very much accentuated and are superimposed on the background flicker as a slow rhythmic change. The change in flicker rate and the side-to-side or rotational movement are specific to the beat condition.

The fluctuations of both brightness and flicker rate apparently arise from alternation of the in-phase and out-of-phase. ${ }^{3}$ The side-to-side movement is analogous to a continual phi-movement where the intervals between the pulses of the two sources favor movement to the left and then change to favor movement to the right (cf. Attneave \& McReynolds, 1950). When this movement combines with the pulsating gamma movement, the lateral movement takes on an elliptical, three-dimensional appearance. Stereoscopic fusion of the two fields by the use of lenses did not affect the perception of movement or its direction.

The purple blotches (Gebhard, 1943) had the appearance of after-images which expanded and contracted with the dark and bright phase of the beat. Of special interest are the color phenomena reported in dichoptic viewing which must have an even more puzzling central origin. These color effects were infrequent and the factor responsible for them was not related to age since the reports were rather evenly distributed over the groups.

These preliminary results suggest that the interaction of repetitive stimuli producing a beat is no different in young children than it is in adults, at least for the frequencies used and up to age 40 . The older Ss did have greater difficulty in perceiving the beat. This difficulty may be due to the known decline of CFF with age. 
The similarities of Ss' reports were more striking than the differences. Of primary importance is that dichoptic beats, which must result from central interaction, can be perceived by most $S$ s and are reported to have the same phenomenal characteristics as binocular beats. By investigating the relation of these dichoptic beats to physical beat frequency, evidence concerning temporal resolution may be obtained (Karrer, 1965, 1967).

\section{REFERENCES}

ATTNEAVE, F., \& McREYNOLDS, P. A visual beat phenomenon. Amer. J. Psychol., 1950, 63, 107-110.

CLAUSEN, J., \& VANDERBILT, C. Visual beats caused by simultaneous electrical and photic flicker. Amer. I. Psychol, 1957, 70, 577-585.

GEBHARD, J. W. Chromatic phenomena produced by intermittent stimulation of the retina. J. exp. Psychol., 1943, 33, 387-406.

KARRER, R. Visual beat phenomena and their relation to the temporal characteristics of perception. Unpublished doctoral dissertation, New School for Social Research, 1965.

KARRER, R. Visual beat phenomena as an index to the temporal characteristics of perception. J. exp. PsychoL, 1967, 75, 372-378.
SCHWARZ, F. Ueber die Binokulare Summation von Flimmerlicht. $Z$. Sinnesphysiol., 1943, 70, 22-29.

von BÉKÉSY, G. Experiments in hearing. New York: McGraw-Hill, 1960.

WEVER, E. G. Beats and related phenomena resulting from the simultaneous sounding of two tones. Psychol. Rev., 1929, 36, 402-418; 512-523.

\section{NOTES}

1. This is an adaptation of part of a doctoral dissertation submitted to the New School for Social Research, 1965. I thank Howard Gruber, Johs Clausen, Jay Bell, and Nancy Karrer.

2. Tables containing the number of Ss perceiving each characteristic may be obtained from the author.

3. The correspondence of slower flicker to in-phase is a function of frequency. At low frequencies faster flicker occurs during out-of-phase if the summative frequency is slow enough not to fuse; the in-phase period, by contrast, gives an appearance of slower "stopped" flicker. Contrarily, at high frequencies, during out-of-phase, the flicker may be fused giving the "stopped" appearance while in-phase comes below fusion giving fast flicker. 